\title{
Compact operators on the weighted Bergman space $A^{1}(\psi)$
}

\author{
by \\ TAO Yu (Jinhua)
}

\begin{abstract}
We show that a bounded linear operator $S$ on the weighted Bergman space $A^{1}(\psi)$ is compact and the predual space $A_{0}(\varphi)$ of $A^{1}(\psi)$ is invariant under $S^{*}$ if and only if $S k_{z} \rightarrow 0$ as $z \rightarrow \partial D$, where $k_{z}$ is the normalized reproducing kernel of $A^{1}(\psi)$. As an application, we give conditions for an operator in the Toeplitz algebra to be compact.
\end{abstract}

1. Introduction. Let $\varphi$ be a positive continuous function on $[0,1)$. We say that $\varphi$ is normal if there exist $0<a<b$ and $r_{0}<1$ such that

$$
\frac{\varphi(r)}{\left(1-r^{2}\right)^{a}} \searrow 0 \quad \text { and } \quad \frac{\varphi(r)}{\left(1-r^{2}\right)^{b}} \nearrow \infty \quad\left(r_{0} \leq r \rightarrow 1^{-}\right) \text {. }
$$

The functions $\{\varphi, \psi\}$ will be called a normal pair if $\varphi$ is normal and if, for some $b$ satisfying (1), there exists $\alpha>b-1$ such that $\varphi(r) \psi(r)=\left(1-r^{2}\right)^{\alpha}$. Let $d A$ denote the normalized Lebesgue measure on the open unit disk $D$ in the complex plane, and $d A_{\psi}$ the measure on $D$ defined by $d A_{\psi}(z)=$ $\psi(|z|) d A(z)$. The condition $\alpha>b-1$ ensures that the measure $d A_{\psi}$ on $D$ is finite.

Let $L^{1}(\psi)$ denote the Banach space of measurable functions $f$ with norm $\|f\|_{\psi}=\int_{D}|f| d A_{\psi}<\infty$. Let $A^{1}(\psi)$ denote the closed subspace of $L^{1}(\psi)$ consisting of all analytic functions, which will be called the weighted Bergman space. In the case that $\varphi(r)=(1-r)^{c}$ for some constant $c>0$, and that $\alpha=c, A^{1}(\psi)$ is the Bergman space $L_{\mathrm{a}}^{1}(D)$.

Let $L_{\infty}(\varphi)$ denote the Banach space consisting of all measurable functions such that $f(z) \varphi(|z|)$ is essentially bounded on $D$ with norm $\|f\|_{\varphi}=$ $\operatorname{ess}_{\sup _{z \in D}}|f(z)| \varphi(|z|)$. Let $A_{\infty}(\varphi)$ denote the closed subspace of $L_{\infty}(\varphi)$ consisting of all analytic functions, and

2000 Mathematics Subject Classification: 47B35, 47A15.

Key words and phrases: weighted Bergman space, compact operator, reproducing kernel, Toeplitz algebra.

Partially supported by the Scientific Research Fund (20040850) of Zhejiang Provincial Education Department of China. 


$$
A_{0}(\varphi):=\left\{f \in A_{\infty}(\varphi): \lim _{r \rightarrow 1^{-}} \sup _{|z|=r} f(z) \varphi(|z|)=0\right\},
$$

a closed subspace of $A_{\infty}(\varphi)$.

Throughout this paper we shall use the following pairing between $A^{1}(\psi)$ and $A_{\infty}(\varphi)$ :

$$
\langle f, g\rangle=\int_{D} f(z) \overline{g(z)}\left(1-|z|^{2}\right)^{\alpha} d A(z)
$$

For $z, w \in D$, let

$$
K_{z}(w)=\frac{1+\alpha}{(1-\bar{z} w)^{2+\alpha}}
$$

and define an operator $Q$ on $L^{1}(\psi)$ by

$$
(Q f)(z)=\left\langle f, K_{z}\right\rangle=\int_{D} f(w) \overline{K_{z}(w)}\left(1-|w|^{2}\right)^{\alpha} d A(w)
$$

Then $Q$ is a bounded projection from $L^{1}(\psi)$ onto $A^{1}(\psi)$ and $K_{z}(w)$ is the reproducing kernel of $A^{1}(\psi)$, that is, $f(z)=\left\langle f, K_{z}\right\rangle$ for any $f \in A^{1}(\psi)$. Since $K_{z}$ is in $A^{1}(\psi)$ for any $z \in D$, the reproducing property $f(z)=\left\langle f, K_{z}\right\rangle$ also holds for $f \in A_{\infty}(\varphi)$ (see [6] for the details). The function $k_{z}(w):=$ $K_{z}(w) /\left\|K_{z}\right\|_{\psi}$ will be called the normalized reproducing kernel of $A^{1}(\psi)$.

In the Bergman space $L_{\mathrm{a}}^{2}(D)$ setting, Axler and Zheng [1] proved that an operator $S$ which is a finite sum of finite products of Toeplitz operators, is compact if and only if $\left\|S k_{z}\right\| \rightarrow 0$ as $|z| \rightarrow 1^{-}$. This result also holds for the spaces $L_{\mathrm{a}}^{p}(D)(1<p<\infty)$ (see $\left.[7]\right), A_{v}^{2}(\Omega)$ with $\Omega$ a regular bounded symmetric domain in $C^{n}$ (see [2]), and $H^{2}(\Omega, d v)$ with $\Omega$ a smoothly bounded multiply connected domain in the complex plane (see [5]). Recently Miao and Zheng [4] proved that for a bounded operator $S$ on $L_{\mathrm{a}}^{p}(D)(1<p<\infty)$ such that both $\sup _{z \in D}\left\|S_{z} 1\right\|_{m}$ and $\sup _{z \in D}\left\|S_{z}^{*} 1\right\|_{m}$ are finite, $S$ is a compact operator on $L_{\mathrm{a}}^{p}(D)$ if and only if the Berezin transform of $S$ tends to 0 at $\partial D$.

In this note, we will obtain a similar result for $A^{1}(\psi)$. More precisely, we show that a bounded linear operator $S$ on $A^{1}(\psi)$ is compact and $A_{0}(\varphi)$ is an invariant subspace of $S^{*}$ if and only if $\left\|S k_{z}\right\|_{\psi} \rightarrow 0$ as $z \rightarrow \partial D$.

2. Preliminaries. The following result can be found in [6].

Lemma 1. $A^{1}(\psi)^{*}=A_{\infty}(\varphi)$ and $A_{0}(\varphi)^{*}=A^{1}(\psi)$.

We also need some results about the reproducing kernel of $A^{1}(\psi)$.

LEMMA 2. There exist constants $c$ and $C$ such that

$$
c / \varphi(|z|) \leq\left\|K_{z}\right\|_{\psi} \leq C / \varphi(|z|) .
$$

Proof. The second inequality can be derived from Lemmas 5 and 8 in [6]. 
Now we prove the first inequality. If $|z| \leq r_{0}$, the first inequality obviously holds. If $|z|>r_{0}$, using (1) and $\varphi(r) \psi(r)=\left(1-r^{2}\right)^{\alpha}$, we have

$$
\begin{aligned}
& \frac{\left(1-|w|^{2}\right)^{\alpha-a}}{\psi(|w|)} \leq \frac{\left(1-|z|^{2}\right)^{\alpha-a}}{\psi(|z|)} \quad \text { when } 1>|w|>|z|, \\
& \frac{\left(1-|w|^{2}\right)^{\alpha-b}}{\psi(|w|)} \leq \frac{\left(1-|z|^{2}\right)^{\alpha-b}}{\psi(|z|)} \quad \text { when } r_{0} \leq|w|<|z| .
\end{aligned}
$$

Hence

$$
\begin{aligned}
&\left\|K_{z}\right\|_{\psi}= \int_{D} \frac{(1+\alpha) \psi(|w|)}{|1-\bar{z} w|^{2+\alpha}} d A(w) \\
& \geq \frac{(1+\alpha) \psi(|z|)}{\left(1-|z|^{2}\right)^{\alpha-b}}\left(\int_{r_{0} \leq|w| \leq|z|} \frac{\left(1-|w|^{2}\right)^{\alpha-b}}{|1-\bar{z} w|^{2+\alpha}} d A(w)\right. \\
&\left.\quad+\int_{|z|<|w|<1} \frac{\left(1-|w|^{2}\right)^{\alpha-a}}{|1-\bar{z} w|^{2+\alpha}\left(1-|z|^{2}\right)^{b-a}} d A(w)\right) .
\end{aligned}
$$

Note that

$$
\frac{1}{\left(1-|z|^{2}\right)^{b-a}}=\frac{1}{((1+|z|)(1-|z|))^{b-a}} \geq \frac{1}{(2|1-\bar{z} w|)^{b-a}},
$$

and similarly

$$
\frac{1}{\left(1-|w|^{2}\right)^{b-a}} \geq \frac{1}{(2|1-\bar{z} w|)^{b-a}} .
$$

So there exists a positive constant $c_{1}$ such that

$$
\begin{aligned}
\left\|K_{z}\right\|_{\psi} & \geq \frac{c_{1} \psi(|z|)}{\left(1-|z|^{2}\right)^{\alpha-b}} \int_{r_{0} \leq|w|} \frac{\left(1-|w|^{2}\right)^{\alpha-a}}{|1-\bar{z} w|^{2+\alpha+b-a}} d A(w) \\
& =\frac{c_{1} \psi(|z|)}{\left(1-|z|^{2}\right)^{\alpha-b}}\left(\int_{D}-\int_{|w| \leq r_{0}}\right) \frac{\left(1-|w|^{2}\right)^{\alpha-a}}{|1-\bar{z} w|^{2+\alpha+b-a}} d A(w) \\
& =: \frac{c_{1} \psi(|z|)}{\left(1-|z|^{2}\right)^{\alpha-b}}\left(I_{1}(z)-I_{2}(z)\right) .
\end{aligned}
$$

Now by Lemma 4.2 .2 of [8], $I_{1}(z) \sim\left(1-|z|^{2}\right)^{-b}$ as $z \rightarrow \partial D$; and $I_{2}$ is bounded. Thus it is easy to see that there exists $c>0$ so that

$$
\left\|K_{z}\right\|_{\psi} \geq c \frac{\psi(|z|)}{\left(1-|z|^{2}\right)^{\alpha}}=c / \varphi(|z|) .
$$

The proof is now complete.

Lemma 3. The normalized reproducing kernel $k_{z}$ converges weakly* to 0 in $A^{1}(\psi)$ as $z \rightarrow \partial D$. 
Proof. For $g \in A_{0}(\varphi)$, by the reproducing property of $K_{z}$, we have

$$
\left\langle k_{z}, g\right\rangle=\frac{\left\langle K_{z}, g\right\rangle}{\left\|K_{z}\right\|_{\psi}}=\frac{\overline{g(z)}}{\left\|K_{z}\right\|_{\psi}} .
$$

Now it follows from the definition of $A_{0}(\varphi)$ and Lemma 2 that $\left\langle k_{z}, g\right\rangle \rightarrow 0$ as $z \rightarrow \partial D$.

\section{Compactness}

Theorem 1. Suppose that $S$ is a bounded linear operator on $A^{1}(\psi)$. Then $S$ is compact and $A_{0}(\varphi)$ is an invariant subspace of $S^{*}$ if and only if $\left\|S k_{z}\right\|_{\psi} \rightarrow 0$ as $z \rightarrow \partial D$.

Proof. Necessity. Suppose that $S$ is a compact operator and $A_{0}(\varphi)$ is an invariant subspace of $S^{*}$. If $\left\|S k_{z}\right\|_{\psi} \nrightarrow 0$ as $z \rightarrow \partial D$, then there exist a constant $\delta>0$ and a sequence $\left\{z_{n}\right\}$ in $D$ such that

$$
z_{n} \rightarrow \partial D \text { and }\left\|S k_{z_{n}}\right\|_{\psi}>\delta .
$$

Since $\left\{k_{z_{n}}\right\}$ is a bounded sequence in $A^{1}(\psi)$ and $S$ is compact, there exists a subsequence of $\left\{k_{z_{n}}\right\}$, also denoted by $\left\{k_{z_{n}}\right\}$, such that $\left\{S k_{z_{n}}\right\}$ converges in $A^{1}(\psi)$. By Lemma $3, z_{n} \rightarrow \partial D$ implies that $k_{z_{n}} \stackrel{w^{*}}{\longrightarrow} 0$. Since $A_{0}(\varphi)$ is an invariant subspace of $S^{*}$, we have, for any $g \in A_{0}(\varphi)$,

$$
\left\langle S k_{z_{n}}, g\right\rangle=\left\langle k_{z_{n}}, S^{*} g\right\rangle \rightarrow 0 .
$$

Thus $S k_{z_{n}} \stackrel{w^{*}}{\longrightarrow} 0$. Since $\left\{S k_{z_{n}}\right\}$ converges in $A^{1}(\psi)$, it must converge to its weak*-limit, that is, 0 . This contradicts (3).

Sufficiency. Suppose that $\left\|S k_{z}\right\|_{\psi} \rightarrow 0$ as $z \rightarrow \partial D$. By the reproducing property of $K_{z}$, one can see that

$$
\left(S^{*} K_{w}\right)(z)=\left\langle S^{*} K_{w}, K_{z}\right\rangle=\overline{\left\langle S K_{z}, K_{w}\right\rangle}=\overline{\left(S K_{z}\right)(w)} .
$$

So for $f \in A^{1}(\psi)$,

$$
\begin{aligned}
(S f)(w) & =\left\langle S f, K_{w}\right\rangle=\left\langle f, S^{*} K_{w}\right\rangle=\int_{D} f(z) \overline{\left(S^{*} K_{w}\right)(z)}\left(1-|z|^{2}\right)^{\alpha} d A(z) \\
& =\int_{D} f(z)\left(S K_{z}\right)(w) \varphi(|z|) d A_{\psi}(z) .
\end{aligned}
$$

For $0<t<1$, define a compact supporting continuous function $\eta_{t}$ on $D$ by

$$
\eta_{t}(z)= \begin{cases}1, & |z| \leq t \\ \frac{1+t}{1-t}-\frac{2|z|}{1-t}, & t<|z| \leq(1+t) / 2 \\ 0, & (1+t) / 2<|z|<1\end{cases}
$$


For any $0<r, t<1$, define the integral operators $S_{[r]}$ on $A^{1}(\psi)$ and $S_{[r, t]}$ from $A^{1}(\psi)$ to $L^{1}(\psi)$ as follows:

$$
\begin{aligned}
S_{[r]} f(w) & =\int_{D} f(z)\left(S K_{z}\right)(w) \eta_{r}(z) \varphi(|z|) d A_{\psi}(z), \\
S_{[r, t]} f(w) & =\int_{D} f(z)\left(S K_{z}\right)(w) \eta_{r}(z) \eta_{t}(w) \varphi(|z|) d A_{\psi}(z) .
\end{aligned}
$$

Using Lemma 2, we have

$$
\begin{aligned}
\left\|\left(S-S_{[r]}\right) f\right\|_{\psi} & \leq \int_{D}|f(z)| d A_{\psi}(z) \int_{D}\left|\left(S K_{z}\right)(w)\right| \varphi(|z|)\left(1-\eta_{r}(z)\right) d A_{\psi}(w) \\
& \leq C\|f\|_{\psi} \sup _{z \in D}\left(1-\eta_{r}(z)\right)\left\|S k_{z}\right\|_{\psi} .
\end{aligned}
$$

Since $\left\|S k_{z}\right\|_{\psi} \rightarrow 0$ as $z \rightarrow \partial D$, we have $\sup _{z \in D}\left(1-\eta_{r}(z)\right)\left\|S k_{z}\right\|_{\psi} \rightarrow 0$ as $r \rightarrow 1^{-}$. Thus

$$
\left\|S-S_{[r]}\right\| \rightarrow 0 \quad \text { as } r \rightarrow 1^{-} .
$$

Seeing $S_{[r]}$ as an operator from $A^{1}(\psi)$ to $L^{1}(\psi)$, if we prove that it is compact, then it is also compact as an operator on $A^{1}(\psi)$. Similar to the above, we have

$$
\left\|S_{[r]}-S_{[r, t]}\right\| \leq C \sup _{z \in \frac{1+r}{2} D} \int_{D}\left|\left(S k_{z}\right)(w)\right|\left(1-\eta_{t}(w)\right) d A_{\psi}(w) .
$$

We will prove that

$$
\sup _{z \in \frac{1+r}{2} D} \int_{D}\left|\left(S k_{z}\right)(w)\right|\left(1-\eta_{t}(w)\right) d A_{\psi}(w) \rightarrow 0
$$

as $t \rightarrow 1^{-}$for fixed $r<1$.

Let $g_{t}(z)=\int_{D}\left|\left(S k_{z}\right)(w)\right|\left(1-\eta_{t}(w)\right) d A_{\psi}(w)$. Firstly we will show that $\left\{g_{t}: 0<t<1\right\}$ is equicontinuous and uniformly bounded on $\frac{\overline{1+r}}{2} D$.

Since the Taylor expansion of $K_{z}$ is $K_{z}(w)=\sum_{m=0}^{\infty} \beta_{m}(w \bar{z})^{m}$, where $\beta_{m}=(\alpha+1) \cdots(\alpha+m+1) / m$ !, for any $z_{1}, z_{2} \in \frac{\overline{1+r}}{2} D$ we have

$$
\begin{aligned}
\left|K_{z_{1}}(w)-K_{z_{2}}(w)\right| & =\left|\sum_{m=0}^{\infty} \beta_{m}\left(w \bar{z}_{1}\right)^{m}-\sum_{m=0}^{\infty} \beta_{m}\left(w \bar{z}_{2}\right)^{m}\right| \\
& \leq \sum_{m=1}^{\infty} \beta_{m}|w|^{m}\left|\bar{z}_{1}^{m}-\bar{z}_{2}^{m}\right| \leq\left|z_{1}-z_{2}\right| \sum_{m=1}^{\infty} \beta_{m} m r^{m-1} .
\end{aligned}
$$

The last series above is the derivative of the series $\sum_{m=0}^{\infty} \beta_{m} r^{m}$ for $\frac{1+\alpha}{(1-r)^{2+\alpha}}$ hence convergent in $|r|<1$. So for any $\varepsilon>0$, there exists a constant $\delta_{1}>0$ such that $\left|K_{z_{1}}(w)-K_{z_{2}}(w)\right| \leq \varepsilon$ for any $z_{1}, z_{2} \in \overline{\frac{1+r}{2} D}$ with $\left|z_{1}-z_{2}\right|<\delta_{1}$, and so $\left\|K_{z}\right\|_{\psi}$ is uniformly continuous on $\frac{\overline{1+r}}{2} D$. Then for any $\varepsilon>0$, there exist 
$\delta_{2}>0$ such that $\left|k_{z_{1}}(w)-k_{z_{2}}(w)\right|<\varepsilon$ for $z_{1}, z_{2} \in \overline{\frac{1+r}{2} D}$ with $\left|z_{1}-z_{2}\right|<\delta_{2}$, whence

$$
\begin{aligned}
\left|g_{t}\left(z_{1}\right)-g_{t}\left(z_{2}\right)\right| & \leq \int_{D}\left|S k_{z_{1}}(w)-S k_{z_{2}}(w)\right|\left(1-\eta_{t}(w)\right) d A_{\psi}(w) \\
& \leq\|S\| \int_{D}\left|k_{z_{1}}(w)-k_{z_{2}}(w)\right| d A_{\psi}(w) \leq \varepsilon\|S\|\|1\|_{\psi} .
\end{aligned}
$$

Since $\varepsilon$ is arbitrary, $\left\{g_{t}: 0<t<1\right\}$ is equicontinuous. It is obvious that $\left\{g_{t}: 0<t<1\right\}$ is uniformly bounded.

For $z \in \overline{\frac{1+r}{2} D}$, Lebesgue's dominated convergence theorem implies that $g_{t}(z) \rightarrow 0$ as $t \rightarrow 1^{-}$. It follows from Ascoli's theorem that $\left\{g_{t}: 0<t<1\right\}$ is relatively compact in $C\left(\frac{\overline{1+r}}{2} D\right)$, the Banach space of continuous functions on $\overline{\frac{1+r}{2} D}$, so has a unique accumulation point, 0 . Therefore $g_{t} \rightarrow 0$ as $t \rightarrow 1^{-}$. So (5) implies that

$$
\left\|S_{[r]}-S_{[r, t]}\right\| \rightarrow 0 \quad \text { as } t \rightarrow 1^{-} .
$$

Since the kernel of $S_{[r, t]}$ is a compact supporting continuous function on $D \times D$, it can be approximated uniformly by polynomials in $z, \bar{z}, w, \bar{w}$ on $D \times D$. Because a polynomial kernel induces a finite rank integral operator, $S_{[r, t]}$ is a compact operator (cf. [3]). Thus (6) implies that $S_{[r]}$ is compact, and (4) implies that $S$ is compact.

Finally, we show that $A_{0}(\varphi)$ is invariant under $S^{*}$. Suppose that $g \in$ $A_{0}(\varphi)$ and $S^{*} g \in A_{\infty}(\varphi) \backslash A_{0}(\varphi)$. By the definitions of $A_{0}(\varphi)$ and $A_{\infty}(\varphi)$, there exist some positive constant $\varepsilon$ and a sequence $\left\{z_{n}\right\}$ in $D$ such that $z_{n} \rightarrow \partial D$ as $n \rightarrow \infty$ and $\left|\left(S^{*} g\right)\left(z_{n}\right)\right| \varphi\left(\left|z_{n}\right|\right)>\varepsilon$. Thus using Lemma 2 and the reproducing property of $K_{z}$, we have

$$
\left|\left\langle S k_{z_{n}}, g\right\rangle\right|=\left|\left\langle k_{z_{n}}, S^{*} g\right\rangle\right|=\frac{\left|\left(S^{*} g\right)\left(z_{n}\right)\right|}{\left\|K_{z_{n}}\right\|_{\psi}} \geq \frac{1}{C}\left|\left(S^{*} g\right)\left(z_{n}\right)\right| \varphi\left(\left|z_{n}\right|\right)>\frac{\varepsilon}{C} .
$$

This contradicts the hypothesis $\left\|S k_{z}\right\|_{\psi} \rightarrow 0$ as $z \rightarrow \partial D$, and completes the proof of Theorem 1 .

In the last argument of the proof of Theorem $1, g \in A_{\infty}(\varphi)$ is only needed. So the following result holds.

COROLlary. If $S$ is a compact operator on $A^{1}(\psi)$ and $S^{*} A_{0}(\varphi) \subset$ $A_{0}(\varphi)$, then $S^{*} A_{\infty}(\varphi) \subset A_{0}(\varphi)$.

REMARK. If $S$ is a compact operator on $L_{\mathrm{a}}^{2}(D)$, then $\left\|S k_{z}\right\| \rightarrow 0$ as $z \rightarrow \partial D$. Examples in [1] and [4] show that the converse does not hold. However, in $A^{1}(\psi),\left\|S k_{z}\right\| \rightarrow 0$ as $z \rightarrow \partial D$ is a sufficient condition for the compactness of $S$. Moreover, the following example shows that $A_{0}(\varphi)$ being an invariant subspace of $S^{*}$ in Theorem 1 is necessary. 
Example. Suppose that $f \in A_{\infty}(\varphi) \backslash A_{0}(\varphi)$. By the definitions of $A_{\infty}(\varphi)$ and $A_{0}(\varphi)$, there exists a sequence $\left\{z_{n}\right\}$ in $D$ such that $z_{n} \rightarrow \partial D$ and $\left|f\left(z_{n}\right)\right| \varphi\left(\left|z_{n}\right|\right) \nrightarrow 0$.

Suppose that $0 \neq g \in A^{1}(\psi)$. Then there exists $h \in A_{\infty}(\varphi)$ such that $\langle g, h\rangle \neq 0$. Let $S=g \otimes f$. Then $S$ is a compact operator on $A^{1}(\psi)$. However,

$$
\begin{aligned}
\left|\left\langle S k_{z}, h\right\rangle\right| & =\left|\left\langle k_{z},(g \otimes f)^{*} h\right\rangle\right|=\left|\left\langle k_{z},\left(f \otimes g^{* *}\right) h\right\rangle\right| \\
& =\left|\left\langle k_{z}, f\right\rangle\langle g, h\rangle\right|=|\langle g, h\rangle| \frac{\left|f\left(z_{n}\right)\right|}{\left\|K_{z_{n}}\right\|_{\psi}} \\
& \geq C|\langle g, h\rangle|\left|f\left(z_{n}\right)\right| \varphi\left(\left|z_{n}\right|\right) \nrightarrow 0,
\end{aligned}
$$

where the inequality comes from Lemma 2 , and $g^{* *}$ denotes the image of $g$ in the double dual space of $A^{1}(\psi)$. So we have $\left\|S k_{z_{n}}\right\|_{\psi} \nrightarrow 0$.

For $u \in L^{\infty}(D)$, define the Toeplitz operator $T_{u}$ by $T_{u}(f)=Q(u f)$, where $f \in A^{1}(\psi)$. Let $\mathcal{T}\left(L^{\infty}\right)$ denote the closed subalgebra of $B\left(A^{1}(\psi)\right)$ generated by $\left\{T_{u}: u \in L^{\infty}(D)\right\}$.

TheOREM 2. Suppose that $S \in \mathcal{T}\left(L^{\infty}\right)$. Then $S$ is compact if and only if $\left\|S k_{z}\right\|_{\psi} \rightarrow 0$ as $z \rightarrow \partial D$.

Proof. By Theorem 1, it is sufficient to prove that $A_{0}(\varphi)$ is invariant under $S^{*}$ when $S \in \mathcal{T}\left(L^{\infty}\right)$. By the definition of $\mathcal{T}\left(L^{\infty}\right)$, it is sufficient to prove that $A_{0}(\varphi)$ is invariant under $T_{u}^{*}$ for any $u \in L^{\infty}(D)$. Let $g \in A_{0}(\varphi)$. Then

$$
\left(T_{u}^{*} g\right)(z)=\overline{\left\langle K_{z}, T_{u}^{*} g\right\rangle}=\overline{\left\langle T_{u} K_{z}, g\right\rangle}=\overline{\left\langle u K_{z}, g\right\rangle} .
$$

Hence

$$
\begin{aligned}
\left|\left(T_{u}^{*} g\right)(z) \varphi(|z|)\right| \leq & \int_{D}\left|u(w) K_{z}(w) g(w)\right|\left(1-|w|^{2}\right)^{\alpha} \varphi(|z|) d A(w) \\
\leq & \|u\|_{\infty} \int_{D} \varphi(|z|)\left|K_{z}(w) g(w)\right| \varphi(|w|) d A_{\psi}(w) \\
= & \|u\|_{\infty}\left(\int_{r D} \varphi(|z|)\left|K_{z}(w) g(w)\right| \varphi(|w|) d A_{\psi}(w)\right. \\
& \left.+\int_{D \backslash r D} \varphi(|z|)\left|K_{z}(w) g(w)\right| \varphi(|w|) d A_{\psi}(w)\right),
\end{aligned}
$$

where $0<r<1$. Since $g \in A_{0}(\varphi)$, for any $\varepsilon>0$, there exists $r$ such that $|g(w)| \varphi(|w|) \leq \varepsilon$ for $w \in D \backslash r D$. Then it follows from Lemma 2 that the second integral on the right hand side of (7) is $\leq \varepsilon \int_{D \backslash r D} \varphi(|z|)\left|K_{z}(w)\right| d A_{\psi}(w)$ $\leq C \varepsilon$ for fixed $r$. Since $g \in A_{0}(\varphi)$, there exists a constant $M$ such that $\sup _{w \in D}|g(w)| \varphi(|w|) \leq M$. Since $\varphi(|z|)\left|K_{z}(w)\right|$ converges uniformly to 0 on $r D$ as $z \rightarrow \partial D$, the first integral on the right hand side of (7) converges to 0 
as $z \rightarrow \partial D$. So we have

$$
\limsup _{z \rightarrow \partial D}\left|\left(T_{u}^{*} g\right)(z)\right| \varphi(|z|) \leq\|u\|_{\infty} C \varepsilon
$$

Since $\varepsilon$ is arbitrary, $\left|\left(T_{u}^{*} g\right)(z)\right| \varphi(|z|) \rightarrow 0$ as $z \rightarrow \partial D$. Thus $T^{*} g \in A_{0}(\varphi)$.

Acknowledgements. The author is grateful to the referee for several comments that improved the paper, in particular indicated the Corollary of Theorem 1.

\section{References}

[1] S. Axler and D. Zheng, Compact operators via the Berezin transform, Indiana Univ. Math. J. 47 (1998), 387-400.

[2] M. Engliš, Compact Toeplitz operators via the Berezin transform on bounded symmetric domains, Integral Equations Operator Theory 33 (1999), 426-455.

[3] P. R. Halmos and V. S. Sunder, Bounded Integral Operators on $L^{2}$-spaces, Springer, Berlin, 1978.

[4] J. Miao and D. Zheng, Compact operators on the Bergman space, Integral Equations Operator Theory 48 (2004), 61-79.

[5] R. Raimondo, Compact operators on the Bergman space of multiply-connected domains, Proc. Amer. Math. Soc. 129 (2000), 739-747.

[6] A. L. Shields and D. L. Williams, Bounded projections, duality, and multipliers in spaces of analytic functions, Trans. Amer. Math. Soc. 162 (1971), 287-303.

[7] T. Yu and S. L. Sun, Berezin transform and Toeplitz operators on $A^{p}(\varphi)$, Acta Anal. Funct. Appl. 2 (2000), no. 1, 73-84 (in Chinese).

[8] K. H. Zhu, Operator Theory in Function Spaces, Dekker, New York, 1990.

Department of Mathematics

Zhejiang Normal University

Jinhua, Zhejiang 321004

P.R. China

E-mail: yufuzhou@zjnu.cn

Received October 28, 2005

Revised version April 28, 2006 